Technical Note

\title{
An In-Orbit Measurement Method for Elevation Antenna Pattern of MEO Synthetic Aperture Radar Based on Nano Calibration Satellite
}

\author{
Tian Qiu ${ }^{1,2}$, Yu Wang ${ }^{1, *}{ }^{\mathbb{D}}$, Jun Hong ${ }^{1}$, Kaichu Xing ${ }^{1,2}$, Shaoyan Du ${ }^{1,2}$ and Jingwen $\mathrm{Mu}^{1,2}$ \\ 1 National Key Laboratory of Science and Technology on Microwave Imaging, Aerospace Information Research \\ Institute, Chinese Academy of Sciences, Beijing 100190, China; qiutian19@mails.ucas.edu.cn (T.Q.); \\ jhong@mail.ie.ac.cn (J.H.); xingkaichu19@mails.ucas.edu.cn (K.X.); dushaoyan17@mails.ucas.edu.cn (S.D.); \\ mujingwen20@mails.ucas.edu.cn (J.M.) \\ 2 University of Chinese Academy of Sciences, Beijing 100049, China \\ * Correspondence: wangyu@mail.ie.ac.cn; Tel.: +86-10-5888-7524
}

\section{check for}

updates

Citation: Qiu, T.; Wang, Y.; Hong, J.;

Xing, K.; Du, S.; Mu, J. An In-Orbit

Measurement Method for Elevation Antenna Pattern of MEO Synthetic Aperture Radar Based on Nano Calibration Satellite. Remote Sens. 2022, 14, 741. https://doi.org/ $10.3390 / \mathrm{rs} 14030741$

Academic Editors: Mi Wang, Hanwen Yu, Jianlai Chen and Ying Zhu

Received: 28 December 2021

Accepted: 3 February 2022

Published: 5 February 2022

Publisher's Note: MDPI stays neutral with regard to jurisdictional claims in published maps and institutional affiliations.

Copyright: (C) 2022 by the authors. Licensee MDPI, Basel, Switzerland. This article is an open access article distributed under the terms and conditions of the Creative Commons Attribution (CC BY) license (https:// creativecommons.org/licenses/by/ $4.0 /)$.

\begin{abstract}
The medium-Earth-orbit synthetic aperture radar (MEO-SAR) is deployed at orbit altitude above low-Earth-orbit synthetic aperture radar (LEO-SAR, around $2000 \mathrm{~km}$ ) and below the geosynchronous orbit SAR (GEO-SAR, near $35786 \mathrm{~km}$ ) to cover a wide swath, which is four to five times larger than LEO-SAR. Therefore, the measurement method for the LEO-SAR elevation antenna pattern using the SAR data acquired over the Amazon tropical rainforest (ground-based method), where the typical width of rainforest area is approximately $150 \mathrm{~km}$, can hardly meet the requirement of a wide swath to determine the MEO-SAR antenna elevation pattern. Moreover, several new MEO-SAR systems are now proposed that will use low frequency, and the low frequency penetration characteristics may affect the elevation antenna pattern determination using homogenous distributed targets such as the Amazon rainforest. This paper proposes a novel space-based method for the in-orbit measurement of the elevation antenna pattern of MEO-SAR based on one nano calibration satellite mounted with a receiver. Through appropriate orbit design, the nano calibration satellite can fly across the entire MEO-SAR swath along the range direction, and the elevation antenna pattern envelope can be extracted from the data recorded by the receiver. Simulation work is performed to verify the feasibility of the proposed space-based method, and the measurement accuracy of this method is analyzed.
\end{abstract}

Keywords: synthetic aperture radar; radiometric calibration; elevation antenna pattern; SAR system calibration; MEO SAR; NanoSat

\section{Introduction}

The medium-Earth-orbit synthetic aperture radar (MEO-SAR) is deployed at orbit altitude above low-Earth-orbit synthetic aperture radar (LEO-SAR, around $2000 \mathrm{~km}$ ) and below the geosynchronous orbit SAR (GEO-SAR, near 35,786 km), which can provide Earth observations that require low spatial resolutions and wide swaths at high temporal resolutions, such as soil moisture estimation, sea ice monitoring and ocean sensing [1,2]. In order to realize the quantitative application of MEO-SAR data and to satisfy the potential use of MEO-SAR, radiometric calibration is essential and the long-term relative radiometric calibration of $0.5 \mathrm{~dB}$ is required [3].

Accurate knowledge of the antenna patterns is of main importance for precise SAR image processing and relative radiometric calibration. The spaceborne SAR antenna patterns are usually tested on the ground before launch. However, it is difficult for large SAR antennas to be measured completely or to simulate the space environment on the ground, and the mechanical structure of antennas may change in orbit due to error factors, such as satellite launch vibration and antenna deployment [4]. Thus, it is essential to obtain 
accurate antenna patterns from in-orbit measurements. The in-orbit measurement of the antenna patterns of the spaceborne SAR is divided into two aspects: azimuth antenna pattern measurement and elevation antenna pattern measurement. The measurement of the SAR azimuth antenna pattern can be realized by using high-precision ground-based receivers [5]: one receiver samples the amplitude of the SAR pulses over time during an overpass, and after transforming the time units to the antenna azimuth angles and correcting by position information, the azimuth antenna pattern can be obtained. By contrast, the in-orbit measurement of the elevation antenna pattern is more complicated because the SAR signals must be sampled over space (swath) to obtain the elevation pattern. Therefore, it is of main importance for SAR radiometric calibration.

In order to realize the quantitative application of SAR data, spaceborne SARs have carried out in-orbit measurements of the elevation antenna pattern. Up until now, three basic approaches of elevation antenna pattern in-orbit measurement are still in use:

(a) Ground receiver method [6-10]. Several ground receivers are deployed in the SAR swaths area along the range direction with certain intervals. Each receiver measures the amplitude of the pulses transmitted by the SAR satellite during an overflight and records the precise time of pulse sampling at the same time. The data recorded by multiple receivers distributed in the range direction can be processed to obtain the elevation antenna pattern. However, multiple ground instruments are required in the case of a wide swath, and thus the workload and cost are very high;

(b) Tropical rainforest method $[4,11]$. The Amazon tropical rainforest is a quite homogeneous scatterer, and thus the shape of the SAR elevation antenna pattern can be clearly visible in the SAR raw data. In order to obtain an accurate two-way elevation antenna pattern, all azimuth lines are summed up and each pixel position is transformed as a function of the elevation angle, through which, the shape of the SAR elevation antenna pattern can be obtained. Due to the restrictions of disturbing areas, the typical width of the Amazon tropical forest area that can be used for calibration is approximately $150 \mathrm{~km}$;

(c) Antenna model method [12-16]. During the 21st century, an active phased array antenna has been widely deployed on spaceborne SAR due to its flexibility in beam forming. As the number of antenna beams increased, the German Aerospace Center (DLR) developed the antenna model method, which derives the antenna patterns from mathematical models in combination with the tropical rainforest method. The application of such an antenna model method has been successfully demonstrated for the TerraSAR-X mission. However, ground-based methods are still required for the verification of the antenna patterns.

The above methods all directly or indirectly use ground-based calibration technology and mainly meet the LEO-SAR antenna pattern measurement requirements. However, the wide-swath characteristic of MEO-SAR brings some challenges to the measurement of the elevation antenna pattern. For example, a MEO-SAR with an orbit altitude of $15000 \mathrm{~km}$, beam pointing angle of $7 \mathrm{deg}$ and range beam width of $2 \mathrm{deg}$ can cover a swath width of approximately $530 \mathrm{~km}$, which is four to five times larger than that of LEO-SAR. The current method based on ground receivers or tropical rain forest (ground-based method) can hardly meet the requirement of a wide swath to determine the MEO-SAR elevation antenna pattern. Furthermore, the MEO-SAR systems tend to have a lower working frequency and stronger signal penetration compared with LEO-SARs [17]. Therefore, the Amazon rainforest may no longer be assumed as homogeneous, as the radar signal is not solely scattered at the canopies of the trees, which makes traditional elevation antenna pattern determination using homogenous distributed targets, such as the Amazon rainforest, inapplicable [18].

The concept of the SAR calibration satellite originated from the calibration satellite for ground-based radar systems $[19,20]$ and the optical calibration standard satellite in space to provide a space-based reference [21-24]. In 2018, the concept of the SAR calibration satellite was introduced and its potential applications were proposed [25]. To cater to a diverse range of missions, the NanoSat and CubeSat have drawn tremendous interest in recent 
years because they enable easy and cost-effective options. In 2011, the US military launched a $3 \mathrm{U}$ CubeSat (Ho'oponopono) developed by the University of Hawaii for calibration requirements of its $80+\mathrm{C}$-band radar tracking stations distributed around the world. Ho'oponopono is the first radar calibration satellite in a CubeSat, which consists of a C-band transponder, GPS unit and associated antennas, all housed in a 3U CubeSat form factor [26]. In addition, a passive SAR using small satellite receivers for persistent Earth observation was proposed $[27,28]$, where the transmitter is a digital television broadcasting station and the receivers are on-board micro-/nano-satellites in low Earth orbit. These provide us with a new thread: Calibration NanoSats or CubeSats working in specially designed orbit are used to carry the calibration instruments, which will equivalently form a moving calibration site in space.

In this paper, we attempt to present a new space-based method for the elevation antenna pattern in-orbit measurement of MEO-SAR based on one nano calibration satellite mounted with a high-precision receiver, which can solve the above problems while achieving an accuracy of $0.3 \mathrm{~dB}$. This method uses the relative motion between the nano calibration satellite and the MEO-SAR satellite, transforming the sampling data over space to sampling data over time (similar to the azimuth antenna pattern measurement using ground receivers). Through appropriate orbit design, the nano calibration satellite, which works in low Earth orbit, can fly across the entire MEO-SAR swath along the range direction. The receiver that is mounted on the nano calibration satellite continuously receives, digitizes and records the SAR signals and, at the same time, the nano calibration satellite and MEO-SAR positions are accurately known. Then, the recording over time can be converted to the MEO-SAR transmit antenna pattern over the elevation angle.

The main innovation and differences between our proposed method and the current methods mentioned above are as follows: (1) The proposed method is a space-based method that can solve the problem of the size of the ground calibration fields not being able to meet the calibration requirements of MEO-SAR. (2) The space-based method can provide a lower cost solution for SAR calibration. Theoretically, one nano calibration satellite mounted with a receiver can realize the measurement of the elevation antenna pattern, whereas multiple ground instruments are required in the ground-based method. (3) The space-based method may provide a solution for GEO-SAR calibration requirements.

This paper is organized as follows: In Section 2, the principle of the proposed spacebased method is introduced. Then, the orbit design method of the nano calibration satellite and all of the error factors of the space-based method are analyzed. Simulation experiments are performed to illustrate the effectiveness of the proposed method in Section 3. Section 4 summarizes the results of experiments with some discussion and research perspectives for the future, and Section 5 gives the conclusions of this paper.

\section{Materials and Methods}

In this section, the elevation antenna pattern measuring principle based on the nano calibration satellite is illustrated first. Then, the details of the calibration activity and the calibration data processing are given. In order to realize the calibration satellite flying across the MEO-SAR beam, the orbit design method of the calibration satellite is proposed. In the following, the error factors that affect the measurement are analyzed.

\subsection{Measuring Principle of The Space-Based Method}

Similar to the normal ground receiver, which measures the SAR azimuth antenna pattern, the space-based method uses the relative motion between the nano calibration satellite and the MEO-SAR satellite, transforming the sampling data over space to sampling data over time. Therefore, for measuring the elevation antenna pattern during a passing of MEO-SAR satellite, as shown in Figure 1, a receiver is mounted on one nano calibration satellite (CAL) working in low Earth orbit, and the CAL should fly across the entire MEOSAR beam along the range direction through appropriate orbit design. The space-based method is mainly composed of two parts: data acquisition and data processing. 
To obtain the calibration data, orbit prediction based on the orbital data of the MEOSAR and the CAL is required for selecting the appropriate CAL-to-SAR calibration arcs, and these operations must be planned in advance. The antenna pointing of the CAL receiver is adjusted to align with the MEO-SAR beam center before each measurement. The receiver deployed on the CAL detects the receive power during the CAL crossing time with an envelope detector, and the detected amplitude is digitized with an analog-to-digital converter. All of the digital values collected during the CAL crossing time are correctly time-labeled and stored within the CAL, which can be transmitted to the ground later.

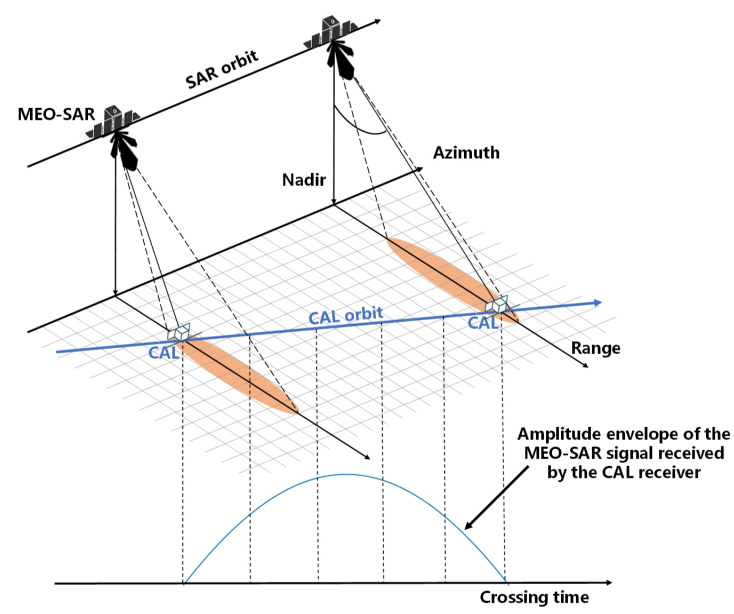

Figure 1. Principle of MEO-SAR elevation antenna pattern measurement using a nano calibration satellite.

After the calibration data are transmitted to the ground, data processing is required to derive the measured antenna pattern from the calibration data. The main steps of the data processing are shown in Figure 2. The first step of the data processing is to transform the recorded digital samples to power expressed in $\mathrm{dB}$ over a time axis. With the known sampling start time, the time axis is determined by GPS time stamps, which were recorded by the CAL receiver in parallel to the receiving power during the crossing time. Due to the relative flight movement of the MEO-SAR and the CAL, the signal recorded by the CAL receiver represents a cut through the antenna pattern along the range direction. Considering the position and velocity information of the MEO-SAR satellite and the CAL determined by GPS units, the time units can be transformed to antenna elevation angles with the known MEO-SAR antenna pointing. After the slant range correction of the receiving power, the data are processed by mean filtering. Then, data profile is normalized and the elevation antenna pattern envelope is obtained by curve fitting.

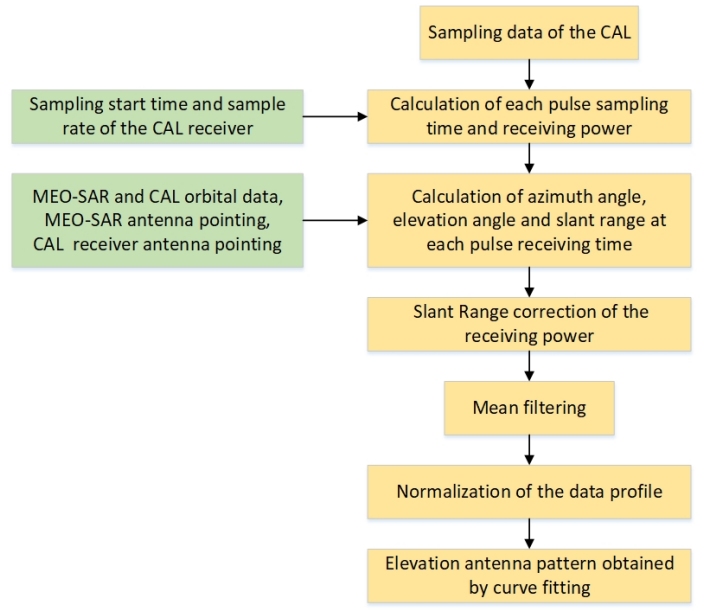

Figure 2. Flowchart of the data processing. 


\subsection{Orbit Design of The Nano Calibration Satellite}

The main purpose of the space-based method is to acquire sampling data of the elevation antenna pattern over time. Therefore, considering the MEO-SAR as the reference object, the CAL should fly across the SAR beam along the range direction at the velocity $v_{c a l_{-} R}$ during the crossing time, as shown in Figure 3; thus, we need to compensate for the azimuth velocity $v_{c a l_{-} A}$ of the CAL, i.e., set $v_{c a l} A=v_{f}$, where $v_{f}$ stands for the velocity of $\overparen{T Q}$. This can be realized by using the relative motion between the MEO-SAR and the CAL through appropriate orbit design of the nano calibration satellite. When the MEO-SAR orbital elements and the orbit altitude of the CAL are known, the orbital element of the CAL that mainly determines the relative motion between the CAL and MEO-SAR is the inclination. Therefore, the main purpose of orbit design is to obtain the inclination of the nano calibration satellite.

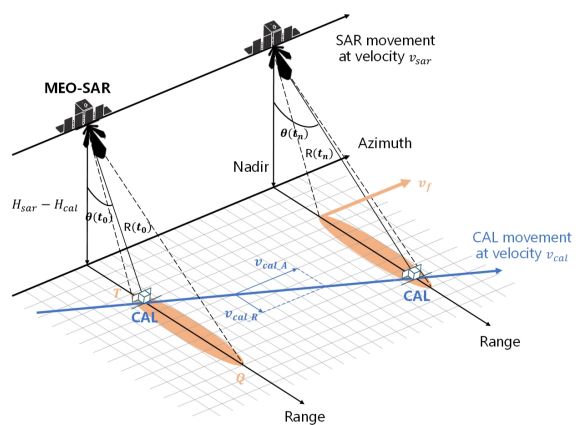

Figure 3. The relative motion between the MEO-SAR and the CAL.

Before the introduction of the orbit design method, analogous to the ground calibration site, the definition of the space calibration site is proposed here. It is assumed that the CAL moves on a sphere with radius $\left(R_{e}+H_{c a l}\right)$, where $R_{e}$ represents the radius of the Earth and $H_{c a l}$ represents the orbit altitude of the CAL. During the crossing time, the range of the MEO-SAR beam irradiated on this spherical surface can be recognized as the space calibration site.

To simplify the analysis, as shown in Figure 4, the CAL and MEO-SAR are assumed to work on circular orbits, and the MEO-SAR orbital elements and the CAL orbit altitude are known. The squint angle of the MEO-SAR antenna beam is 0 deg and the beam center angle is $\theta_{l}$, where $S P$ represents the MEO-SAR beam center. In Figure $4, \widehat{T Q}$ stands for the coverage scope of the space beam footprint in the space calibration site along the range direction and $\overparen{R M}$ stands for ground beam footprint. Firstly, the velocity of MEO-SAR is calculated as Equation (1):

$$
v_{\text {sar }}=\sqrt{\frac{\mu}{a_{\text {sar }}\left(1-e_{\text {sar }}^{2}\right)}}
$$

where $a_{\text {sar }}$ represents the semimajor axis of the MEO-SAR satellite, $e_{s a r}$ represents the eccentricity of the MEO-SAR satellite, which is 0 here, and $\mu$ represents the geocentric gravitational constant. Similarly, we can calculate the velocity of the nano calibration satellite $\left(v_{c a l}\right)$. Therefore, the velocity of any point on $\overparen{T Q}$ is defined, according to RK Raney, [29] as Equation (2):

$$
v_{p}=v_{s a r} \cdot \frac{R_{e}+H_{c a l}}{R_{e}+H_{s a r}} \cdot \cos \left[\arcsin \left(\frac{R_{i n c}}{R_{e}+H_{\text {sar }}} \cdot \sin \theta_{\text {inc }}\right)\right]
$$

where $R_{i n c}$ is the slant range from the MEO-SAR to point on $\overparen{T Q}, \theta_{i n c}$ is the incident angle of each point and $H_{\text {sar }}$ is the orbit altitude of the MEO-SAR. It is clear that the velocity of 
different points on $\overparen{T Q}$ is not the same. Therefore, the velocity of the center $P$ of the space beam footprint is chosen to represent the velocity of $\overparen{T Q}$ as Equation (3):

$$
v_{f}=v_{\text {sar }} \cdot \frac{R_{e}+H_{c a l}}{R_{e}+H_{s a r}} \cdot \cos \left[\arcsin \left(\frac{R_{0}}{R_{e}+H_{s a r}} \cdot \sin \theta_{i}\right)\right]
$$

where $R_{0}=S P$, and due to the large orbital height difference between MEO-SAR and CAL, the spherical curvature of $\overparen{T Q}$ must be considered. Therefore, the line of sight (LOS) angle $\theta_{l}$ is not equal to the incident angle $\theta_{i}$, as shown in Figure 4 , and thus $R_{0}$ is calculated as Equation (4):

$$
R_{0}=\left(R_{e}+H_{s a r}\right) \cdot \frac{\sin \left(\theta_{i}-\theta_{l}\right)}{\sin \theta_{i}}
$$

According to the law of sines, in $\triangle S O P$,

$$
\sin \theta_{i}=\frac{R_{e}+H_{\text {sar }}}{R_{e}+H_{\text {cal }}} \cdot \sin \theta_{l}
$$

Therefore, $v_{f}$ is calculated as Equation (6):

$$
v_{f}=v_{s a r} \cdot \frac{R_{e}+H_{c a l}}{R_{e}+H_{s a r}} \cdot \cos \left(\theta_{i}-\theta_{l}\right)
$$

Then, set the azimuth velocity of the CAL $v_{c a l_{-} A}=v_{f}$, and the inclination of the CAL is calculated as Equation (7):

$$
i_{c a l}=i_{\text {sar }}+\arccos \left(v_{c a l-A} / v_{c a l}\right)
$$

where $i_{\text {sar }}$ represents the inclination of MEO-SAR. Therefore, the inclination $i_{c a l}$ of the CAL can be acquired through the above steps.

However, the above orbit design method faces a problem where the velocity of each point on $\widehat{T Q}$ is not equal, and the velocity of the $P$ point is chosen to represent the velocity of $\widehat{T Q}$ for further calculation. This approximation can introduce a problem where the CAL azimuth velocity $v_{c a l} A$ is not completely compensated during the crossing time. Therefore, the trajectory of the CAL is not a straight line along the range direction, which may introduce azimuth pattern coupling error to the elevation antenna pattern measurement. In subsequent sections, this part of error will be further analyzed.

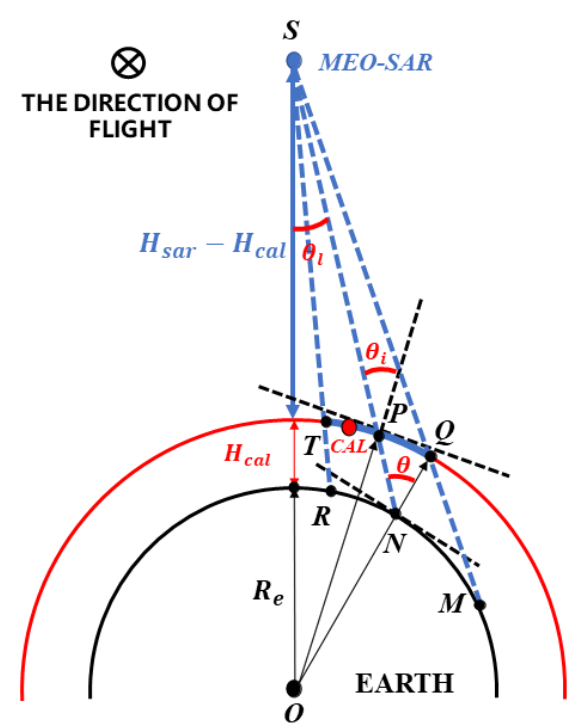

Figure 4. Orbital geometry of MEO-SAR and CAL viewed in section along the MEO-SAR velocity vectors. 


\subsection{Error Factors of the Space-Based Method}

Theoretically, the ideal MEO-SAR signal power $P_{R}(t)$ received by the CAL receiver at the sampling time $t$ can be expressed as follows:

$$
P_{R}(t)=P_{T} \cdot G_{T}(\theta(t), 0) \cdot \frac{g_{R} \cdot G_{R}\left(\theta_{R}(t), \phi_{R}(t)\right) \cdot \lambda^{2}}{(4 \pi R(t))^{2}}
$$

where $P_{T}$ is the SAR transmitting power, $G_{T}(\theta(t), 0)$ is the ideal SAR transmit elevation antenna gain, $g_{R}$ is the CAL receiver channel gain, $G_{R}\left(\theta_{R}(t), \phi_{R}(t)\right)$ is the CAL receiver antenna gain, $\lambda$ is the radar wavelength and $R(t)$ is the slant range from the MEO-SAR satellite to the CAL receiver at the sampling time. Based on the analysis above, the orbit design method of the CAL can introduce azimuth angle offset $\phi(t)$ due to the approximate calculations, and the azimuth angle offset $\phi(t)$ can be calculated and predicted from the known orbital elements of the MEO-SAR and the CAL. The SAR transmit elevation antenna gain in Equation (8) is $G_{T}(\theta(t), \phi(t))$ instead of $G_{T}(\theta(t), 0)$. Therefore, the measured elevation antenna pattern obtained by the CAL receiver is azimuth coupling and expressed as:

$$
G_{T}(\theta(t), \phi(t))=P_{T} \cdot \frac{g_{R} \cdot G_{R}\left(\theta_{R}(t), \phi_{R}(t)\right) \cdot \lambda^{2}}{(4 \pi R(t))^{2} \cdot P_{R}(t)}
$$

Similar to the normal ground receiver, which measures the SAR antenna azimuth pattern, the space-based method uses the relative motion between the nano calibration satellite and the MEO-SAR satellite, transforming the sampling data over space to sampling data over time. Therefore, the error factors analysis of the space-based method can be analogous to the ground receiver method. However, the difference between these two methods must be considered:

- Different measuring duration. Measuring time of the ground receiver method depends on the synthetic aperture time. In this space-based method, the measuring duration of the elevation antenna pattern is mainly determined by the range of $\overparen{T Q}$ and the velocity component $v_{\mathrm{Cal}_{-} \mathrm{R}}$ of the calibration satellite along the range direction, which is much longer compared to the ground-based method;

- Different motion of receiver. The ground receivers are deployed on the calibration site with fixed location measured by ground GPS devices, and the receiver antenna is precisely pointing to the SAR satellite according to local incident angle. However, the receiver mounted on the CAL is always in motion during the crossing time, and the antenna pointing is adjusted before each measurement and will not change during the crossing time;

- Different measuring environment. Since the ground receivers are deployed on the ground calibration sites, the background clutter may affect the antenna pattern measurement results. However, the measuring environment of the CAL receiver is in space, and the influence of the background clutter can be ignored.

Considering the difference between the space-based method and ground receiver method, the following error factors exist in the elevation antenna pattern measurement based on Equation (9):

- $\quad$ SAR transmitting power stability $\sigma_{P_{T}}$ : As the measurement time elapses, SAR transmitting power changes due to the temperature drift of SAR instrument, which can directly affect the amplitude of the SAR signal and the antenna pattern measurement. Due to the longer measurement duration compared with the ground receiver method, the influence of the SAR transmitting power stability $\sigma_{P_{T}}$ on each sampling point should be taken into consideration in this space-based method. The characteristics of SAR transmitting power stability can be measured and corrected through SAR internal calibration. Therefore, in the subsequent simulation, the SAR transmitting power stability $\sigma_{P_{T}}$ can refer to the internal calibration accuracy; 
- The receiver channel gain stability $\sigma_{g_{R}}$ : Low amplitude variation of the receiving power is important for the elevation antenna pattern measurement, and the module that mainly affects the channel gain in the receiver is the low noise amplifier (LNA). Similar to the SAR transmitting power stability, the receiver channel gain stability $\sigma_{g_{R}}$ (i.e., the gain stability of the LNA) is affected by the temperature drift as well, and the receiver channel gain calibration is required to reduce the influence of its impact on the antenna pattern measurement;

- $\quad$ SAR-to-CAL range measurement error $\sigma_{R}$ : The MEO-SAR and CAL use GPS units to achieve precise orbit determination. The SAR-to-CAL slant range measurement error is introduced by the GPS orbit determination errors (random error). Although less accurate than position determination of ground receiver, the ratio of slant range measurement error $\sigma_{R}$ is very small and the resulting error can be ignored due to the large difference in orbit altitudes between the CAL and the MEO-SAR;

- MEO-SAR antenna pointing error: The MEO-SAR satellites use star sensors to determine the satellite's three-axis attitude. The MEO-SAR attitude errors can lead to antenna pointing errors ( $\sigma_{\theta_{P}}$ in elevation and $\sigma_{\phi_{P}}$ in azimuth) and are mainly composed of the systematic bias (due to errors in the sensor installation) and the random measurement error (related to the measurement accuracy of the star sensors);

- CAL receiver antenna pointing error: In order to reduce costs and power consumption, nano calibration satellites generally use sun sensors or gyroscopes to determine threeaxis attitudes (lower accuracy compared with the star sensor). Similarly, the CAL attitude errors can lead to CAL receiver antenna pointing errors $\left(\sigma_{\theta_{R}}\right.$ and $\left.\sigma_{\phi_{R}}\right)$, which can cause the CAL receiver antenna gain error. In addition, the pointing of the receiver antenna continuously changes with the movement of the CAL. Therefore, the $3 \mathrm{~dB}$ beam-width of the antenna should be wide enough (not less than $20 \mathrm{deg}$ ), and so pointing accuracy and stability requirements are not too rigorous;

- The signal-to-noise ratio (SNR): Since the ground receivers are deployed on the ground calibration sites, the background clutter may affect the signal-to-noise ratio, which, in turn, affects antenna pattern measurement results. Therefore, appropriate calibration sites must be considered to improve the SNR and reduce the influence of the background clutter on the antenna pattern measurement. However, the measuring environment of the CAL receiver is in space, and the measurement error caused by the signal-to-noise ratio can be ignored.

The above error factors are independent of each other. Therefore, the measurement accuracy of the elevation antenna pattern considering all of the error factors above can be expressed as follows:

$$
\sigma_{G_{T}}=\sqrt{\left(\frac{\partial G_{T}}{\partial P_{T}}\right)^{2} \sigma_{P_{T}}+\left(\frac{\partial G_{T}}{\partial g_{R}}\right)^{2} \sigma_{g_{R}}+\left(\frac{\partial G_{T}}{\partial R}\right)^{2} \sigma_{R}+\left(\frac{\partial G_{T}}{\partial \theta_{T}}\right)^{2} \sigma_{\theta_{T}}+\left(\frac{\partial G_{T}}{\partial \phi_{T}}\right)^{2} \sigma_{\phi_{T}}+\left(\frac{\partial G_{T}}{\partial \theta_{R}}\right)^{2} \sigma_{\theta_{R}}+\left(\frac{\partial G_{T}}{\partial \phi_{R}}\right)^{2} \sigma_{\phi_{R}}}
$$

\section{Results and Analysis}

In this section, the simulation experiments are performed to validate the proposed space-based method. The simulation process and the simulation parameters are given in Sections 3.1 and 3.2. To verify the feasibility of the orbit design method, simulations of orbital motion during a period of one year have been performed to examine calibration opportunities between the MEO-SAR and the CAL in Section 3.3. Then, the performance of the space-based method is presented in Section 3.4 by comparing the difference between the measured elevation antenna pattern and the reference antenna pattern.

\subsection{Simulation Process}

The main processing flow of the simulation experiment is shown in Figure 5 and the detailed steps are described as follows. 


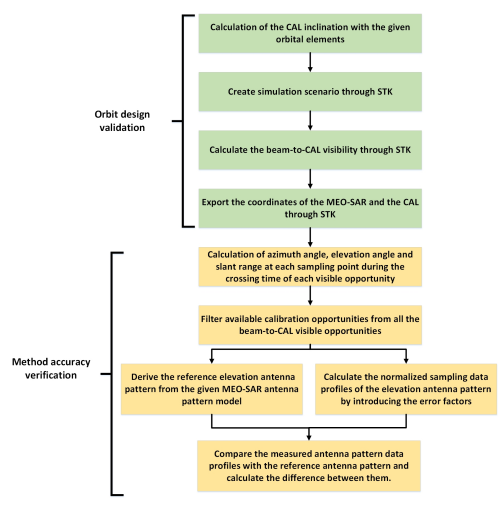

Figure 5. Flowchart of the simulation process.

(1) With the given orbital elements of the MEO-SAR and the orbit altitude of the CAL, calculate the inclination of the CAL through the orbit design method in Section 2;

(2) With the known orbital elements of the MEO-SAR and the CAL, create a scenario containing the MEO-SAR and the CAL through Satellite Tool Kit (STK);

(3) With the current MEO-SAR beam parameters, include the boresight and elevation beamwidth, and calculate the beam-to-CAL visibility (include visible start time, stop time and duration) in the analysis period of the scenario through STK;

(4) Export the coordinates and velocity of the MEO-SAR and the CAL in the inertial coordinate system of each beam-to-CAL visible opportunity at a fixed time step size through STK;

(5) With the known coordinates and velocity of the MEO-SAR and the CAL, calculate the azimuth angle, elevation angle and the SAR-to-CAL slant range at each sampling point during the crossing time of each beam-to-CAL visible opportunity considering the system parameters and the imaging geometry;

(6) To screen the calibration opportunity from all visible opportunities, the following condition is applied: for each visible opportunity, if its range of elevation angle can cover the current SAR beam's elevation beamwidth, this opportunity can be regarded as a calibration opportunity for antenna pattern measurement;

(7) Derive the reference elevation antenna pattern from the given MEO-SAR antenna pattern model;

(8) With the given MEO-SAR antenna pattern model, the calculated results in (5) and the system parameters, based on Equation (8), the measured MEO-SAR signal power (in $\mathrm{dB}$ ) received by the CAL receiver of each sampling point can be calculated by introducing the error factors analyzed in Section 2.3. Based on the data processing steps in Figure 2, the normalized sampling data profiles of the elevation antenna pattern can be calculated and the fit of the sampling data profiles can be obtained through polynomial curve fitting;

(9) Compare the measured antenna pattern data profiles calculated in (8) with the reference antenna pattern obtained in (7) and calculate the difference between them. Then, the fit of the difference can be obtained through polynomial curve fitting.

\subsection{Simulation Parameters and Error Factors}

To facilitate the above simulation steps, a horn antenna is deployed on the CAL receiver and the MEO-SAR transmit antenna pattern is expressed as:

$$
G(\theta, \phi)=\sin c^{2}\left(\frac{\pi \cdot D_{R} \sin \theta}{\lambda}\right) \cdot \sin c^{2}\left(\frac{\pi \cdot D_{A} \sin \phi}{\lambda}\right)
$$

where $D_{R}$ and $D_{A}$ are the antenna length in elevation and azimuth, respectively, $\theta$ is the elevation angle and $\phi$ is the azimuth angle. The main mission and instrument parameters of the MEO-SAR and the CAL used to carry out the elevation antenna pattern measurement performance analysis are shown in Table 1 . Reference parameters for the simulation of the 
error factors is presented in Table 2. The SAR transmitting power stability and receiver channel gain stability refer to the internal calibration accuracy of the TerraSAR-X [12]. The poor orbit determination accuracy by GPS is chosen to indicate the little effect on the measurement. Since the systematic bias caused by the errors in the sensor installation can be corrected, the random attitude measurement errors are mainly considered in the following simulation.

Table 1. The main mission and instrument parameters used to carry out the elevation antenna pattern measurement performance analysis.

\begin{tabular}{cc}
\hline MEO-SAR Orbit Parameters & Values \\
\hline Orbit Altitude & $15,000 \mathrm{~km}$ \\
Semimajor Axis & $21,371.393 \mathrm{~km}$ \\
Eccentricity & 0 \\
Inclination & $98 \mathrm{deg}$ \\
\hline Radar Parameters & Values \\
\hline Radar Center Frequency & $5.4 \mathrm{GHz}$ \\
$D_{A}$ & $4 \mathrm{~m}$ \\
$D_{R}$ & $0.7 \mathrm{~m}$ \\
Look Direction & $\mathrm{R}$ \\
Squint Angle & $0 \mathrm{deg}$ \\
Center Look Angle & $7 \mathrm{deg}$ \\
Azimuth Beam Width & $0.25 \mathrm{deg}$ \\
Range Beam Width & $1 \mathrm{deg}$ \\
Equivalent Isotropically Radiated Power (EIRP) & $45 \mathrm{dBW}$ \\
\hline CAL Receiver Parameters & Values \\
\hline Antenna Gain & $10 \mathrm{~dB}$ \\
Receiving Pattern 3 dB Beam Width & $30 \mathrm{deg}$ \\
\hline
\end{tabular}

Table 2. Reference parameters for the simulation of the error factors.

\begin{tabular}{cc}
\hline Error Factors & Values \\
\hline SAR Transmitting Power Stability & $0.3 \mathrm{~dB}(3 \sigma)$ \\
The Receiver Channel Gain Stability & $0.2 \mathrm{~dB}(3 \sigma)$ \\
Orbit Determination Accuracy by GPS & $60 \mathrm{~m}(3 \sigma)$ \\
Three-Axis Attitude Accuracy of the MEO-SAR & $0.01 \mathrm{deg}(3 \sigma)$ \\
Three-Axis Attitude Accuracy of the CAL & $0.15 \mathrm{deg}(3 \sigma)$ \\
\hline
\end{tabular}

\subsection{Obit Design Validation}

With the given orbit parameters of the MEO-SAR presented in Table 1, when the orbit altitude of the CAL is $800 \mathrm{~km}$, the inclination of the CAL is calculated as $177.14 \mathrm{deg}$ through the orbit design method in Section 2. To analyze the appropriate CAL-to-SAR calibration opportunities, a scenario containing the MEO-SAR and the CAL is created through STK, as shown in Figure 6. The analysis period of the scenario is one year, of which, the analysis start time and stop time are 16 Nov 2021 04:00:00.000 UTCG and 17 Nov 2022 04:00:00.000 UTCG, respectively. The initial orbital elements of the MEO-SAR satellite and the CAL at the analysis start time of the scenario is presented in Table 3.

By imposing the MEO-SAR beam parameters and SAR-to-CAL slant range constraints, simulations of orbital motion during a period of one year based on the above parameters have been performed to examine the beam-to-CAL visibility through STK, which include the visible start time, stop time and duration of each visible opportunity. The beam-to-CAL visible opportunities are depicted in Table 4, which illustrates that there are 15 beam-to-CAL visible opportunities within a year. However, not all of the visible opportunities obtained 
by STK can be used for the elevation antenna pattern measurement, and the condition applied here to determine whether the visible opportunity can be used for calibration is that the initial and final values of the elevation angle of the visible opportunity should correspond to the SAR satellite beam parameters (step (6) of the simulation process).

Therefore, for verifying the feasibility of the above opportunities, the coordinates of the MEO-SAR and the CAL in the inertial coordinates of each opportunities are exported (with a time step size of $0.01 \mathrm{~s}$ ) for the calculation of the azimuth angle and elevation angle. With the known position and velocity information of the MEO-SAR and the CAL during the crossing time, the azimuth angle, elevation angle and SAR-to-CAL slant range at each sampling point are calculated. The visible opportunity 15 in Table 4 does not meet the condition; thus, it cannot be used for antenna pattern measurement. Figure 7 illustrates the elevation angle over the crossing time of opportunity 1-14, which demonstrates that the range of elevation angle can cover the current SAR beam's elevation beamwidth. Therefore, 14 calibration opportunities are available for the antenna pattern measurement, which indicates that the orbit design method is feasible.

Table 3. Initial orbital elements of the MEO-SAR satellite and the CAL at the start time of the scenario.

\begin{tabular}{ccccccc}
\hline Satellite & Semimajor Axis & Eccentricity & Inclination & Argument of Perigee & RAAN & True Anomaly \\
\hline MEO-SAR & $21,371.393 \mathrm{~km}$ & $0 \mathrm{deg}$ & $98 \mathrm{deg}$ & $270 \mathrm{deg}$ & $88 \mathrm{deg}$ & $0 \mathrm{deg}$ \\
CAL & $7171.393 \mathrm{~km}$ & $0 \mathrm{deg}$ & $177.14 \mathrm{deg}$ & $270 \mathrm{deg}$ & $88 \mathrm{deg}$ & $0 \mathrm{deg}$ \\
\hline
\end{tabular}

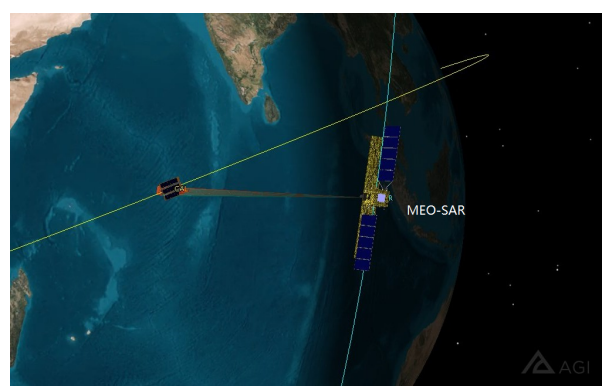

Figure 6. Sketch of the MEO-SAR and CAL in the created scenario.

Table 4. Elevation antenna pattern measurement opportunities within one year.

\begin{tabular}{cccc}
\hline Opportunities & Start Time (UTCG) & Stop Time (UTCG) & Duration (s) \\
\hline 1 & 23 November 2021 15:16:50.779 & 23 November 2021 15:17:28.002 & 37.223 \\
2 & 9 January 2022 22:53:30.453 & 9 January 2022 22:54:07.677 & 37.224 \\
3 & 24 January 2022 21:27:49.336 & 24 January 2022 21:28:26.557 & 37.221 \\
4 & 8 February 2022 19:45:49.781 & 8 February 2022 19:46:27.005 & 37.224 \\
5 & 13 March 2022 05:04:29.005 & 13 March 2022 05:05:06.229 & 37.224 \\
6 & 28 March 2022 03:38:47.889 & 28 March 2022 03:39:25.110 & 37.221 \\
7 & 12 April 2022 01:56:48.333 & 12 April 2022 01:57:25.557 & 37.223 \\
8 & 14 May 2022 11:15:27.557 & 14 May 2022 11:16:04.782 & 37.225 \\
9 & 29 May 2022 09:49:46.441 & 29 May 2022 09:50:23.663 & 37.221 \\
10 & 13 June 2022 08:07:46.887 & 13 June 2022 08:08:24.111 & 37.224 \\
11 & 30 July 2022 16:00:44.994 & 30 July 2022 16:01:22.215 & 37.221 \\
12 & 14 August 2022 14:18:45.439 & 14 August 2022 14:19:22.662 & 37.223 \\
13 & 30 September 2022 22:11:43.547 & 30 September 2022 22:12:20.767 & 37.220 \\
14 & 15 October 2022 20:29:43.991 & 15 October 2022 20:30:21.215 & 37.224 \\
15 & 8 December 2021 13:35:03.366 & 8 December 2021 13:35:28.451 & 25.085 \\
\hline
\end{tabular}

Ideally, the azimuth angle $\phi(t)$ in Equation (9) as the CAL flies across the MEO-SAR beam should be 0 deg. However, Figure 8 illustrates the azimuth angle variation over the crossing time of CAL in the MEO-SAR beam of each calibration opportunity. It can be seen that the CAL can fly across the MEO-SAR beam along the range direction at each opportunity, but with different azimuth angle offsets due to the approximation in 
the orbit design method, as analyzed before. Among them, the azimuth angle offset of opportunity 8 is the largest, and the offset range is $0.112-0.120 \mathrm{deg}$. The azimuth angle offset of opportunity 9 is the smallest, and the offset range is $0-0.007 \mathrm{deg}$. Therefore, the elevation antenna pattern measurement can be realized by the CAL, of which, the orbital elements are designed by the above method, but, for some opportunities, the azimuth pattern coupling error brought about by the method itself cannot be ignored.

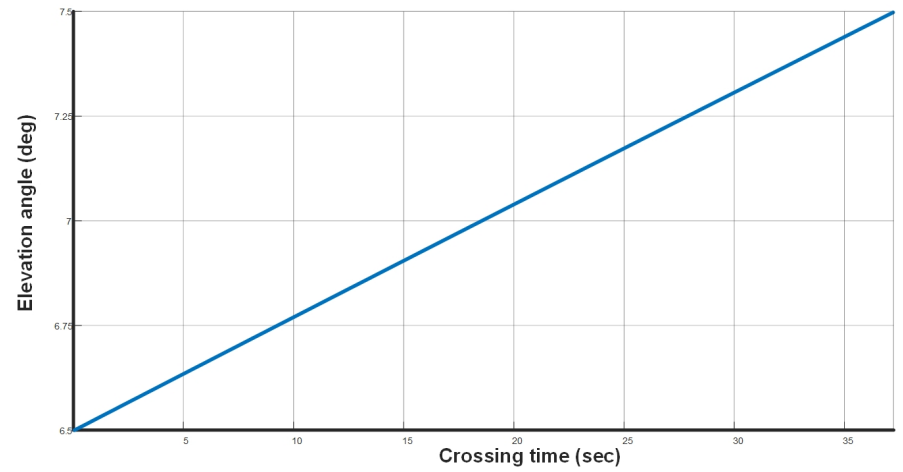

Figure 7. Elevation angle over time of each opportunity during the crossing time.

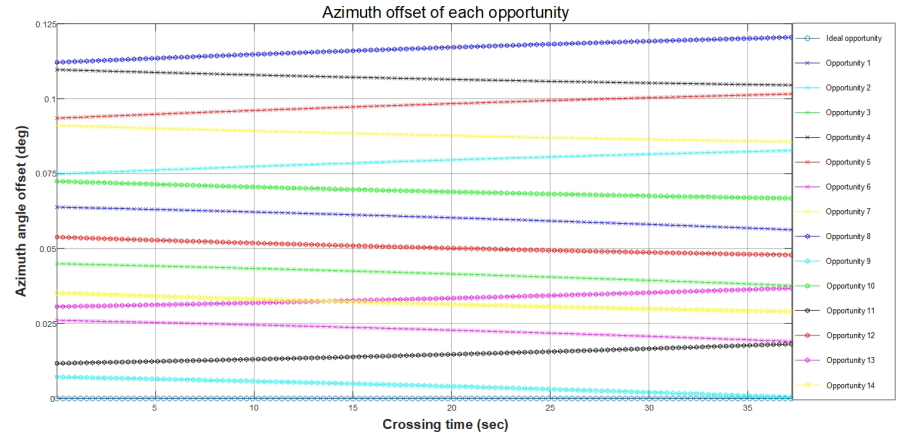

Figure 8. Azimuth angle offset of CAL in the MEO-SAR beam of each opportunity.

\subsection{Method Accuracy Verification}

In this part, elevation antenna pattern measurement simulation experiments are conducted to evaluate the performance of the space-based method by comparing the measured antenna pattern with the reference antenna pattern. The main mission and instrument parameters of the MEO-SAR and CAL are presented in Table 1. According to step (7) of the simulation process, the reference elevation antenna pattern is derived from the given antenna model expressed as Equation (11), where the azimuth angle $\phi(t)=0$ during the crossing time (with no azimuth angle offset). By introducing the error factors listed in Table 2, all of which are Gaussian errors with mean values of 0 and are independent of each other, the opportunity 8 (with the maximum azimuth angle offset) and opportunity 9 (with the minimum azimuth angle offset) are chosen for the elevation antenna pattern measurement.

The reference antenna pattern and the measurement results within the $3 \mathrm{~dB}$ beamwidth can be obtained through steps (7)-(8) of the simulation process, as shown in Figure 9. Figure $9 a, c$ illustrate the comparison between (green points) the antenna pattern sampling data profiles of opportunity 8 and opportunity 9 acquired from the CAL receiver and (blue line) the reference elevation pattern. In addition, (red line in Figure 9a,c) the fit of the sampling data profiles is acquired by polynomial curve fitting of the (green points) antenna pattern sampling data profiles. It can be seen that the verification of the space-based method shows a good accordance between the measured antenna pattern data profiles and the reference antenna pattern. Figure $9 b, d$ illustrate the difference between the reference pattern and sampling data profiles of opportunity 8 and opportunity 9 , and the red line is a 
fit of this difference. It is clear that the deviation between the reference antenna pattern and the measurements for both shapes within the main beam is small. Even the deviation of opportunity 8 , which has the maximum azimuth angle offset, is kept under $0.3 \mathrm{~dB}$ along the $3 \mathrm{~dB}$ region of the elevation patterns. Therefore, the deviation and, hence, the accuracy of the space-based method are within $0.3 \mathrm{~dB}$, which also shows the effectiveness of the space-based method.

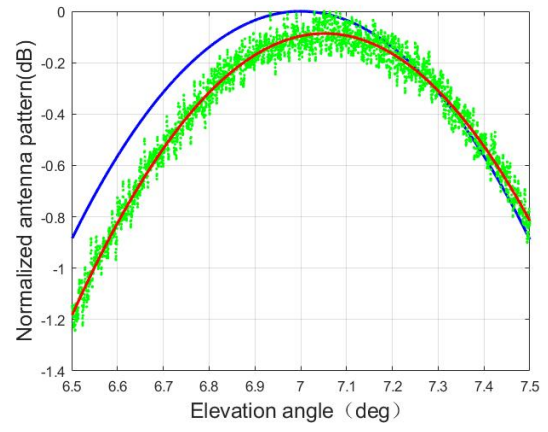

(a) Opportunity 8

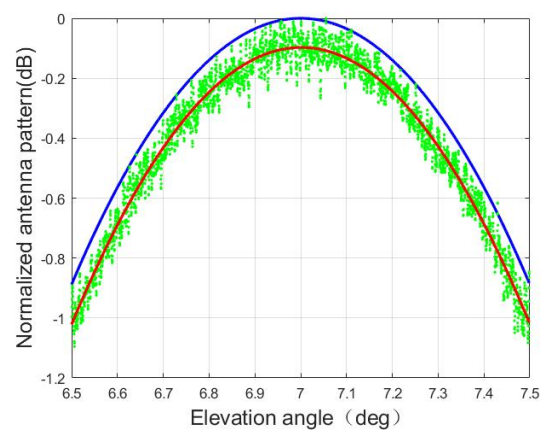

(c) Opportunity 9

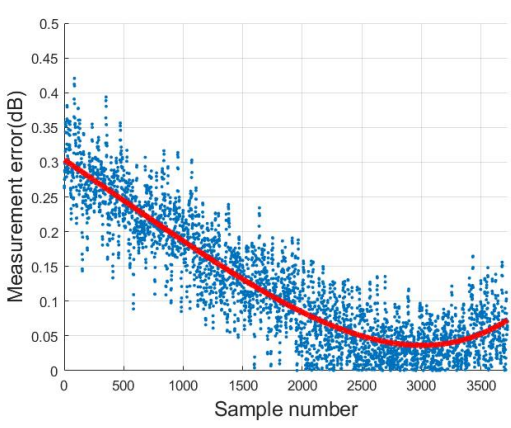

(b) Opportunity 8

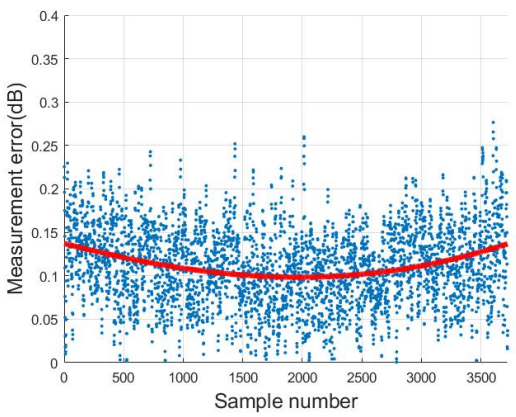

(d) Opportunity 9

Figure 9. (a,c) Comparison between (green points) sampling data profiles of opportunity 8 and opportunity 9 acquired from the CAL receiver and (blue line) reference elevation pattern; the red line is a fit of the sampling data profiles. $(\mathbf{b}, \mathbf{d})$ Difference between reference pattern and sampling data profiles of opportunity 8 and opportunity 9 ; the red line is a fit of this difference.

\section{Discussion}

In this paper, we have described a new space-based method for MEO-SAR elevation antenna pattern measurement using one nano calibration satellite mounted with a receiver. In order to obtain the sampling data over time of the elevation antenna pattern, the basic orbit design method of the nano calibration satellite is introduced and the error factors that affect the elevation antenna pattern measurement are analyzed. Then, we have performed a comprehensive set of simulations to validate the proposed space-based method. The results of this study show that the proposed space-based method is feasible and that the measurement accuracy of the MEO-SAR elevation antenna pattern is within $0.3 \mathrm{~dB}$, which can meet the current antenna pattern measurement accuracy requirements. Compared with the ground-based method, the proposed space-based method moves the calibration site from ground to space, which can break through the limitation of the size of the ground calibration site. In addition, with the known orbital elements of the SAR and nano calibration satellite, the appropriate calibration opportunities for the spaceborne SAR can be predicted and the nano calibration satellite can provide unattended and low-cost calibration solutions for the spaceborne SAR.

Our results suggest a possibility of an in-orbit measurement for the MEO-SAR elevation antenna pattern based on a nano calibration satellite. However, the orbit design method in this paper is primary and analyzed at the principle level. To simplify the analysis, 
the orbit types of MEO-SAR and CAL in this paper are assumed to be a circular orbit. The specific orbit design method of the nano calibration satellite for other orbit types is not discussed here. In the future work, the orbit constraint and the optimization of the nano calibration satellite will be discussed in combination with the specific MEO-SAR orbit parameters. In addition, the hardware implementation of the CAL receiver and the nano calibration satellite system will be analyzed.

\section{Conclusions}

The in-orbit measurement for the elevation antenna pattern of MEO-SAR faces the challenge of the ground-based method being unavailable due to the wide swath of MEOSAR. This paper proposes a novel space-based method that uses one nano calibration satellite mounted with a receiver for MEO-SAR elevation antenna pattern measurement, which is based on the fact that receivers can receive the sampling data of the pulse signals transmitted from the MEO-SAR. In order to obtain the antenna pattern sampling data over time along the range direction using a nano calibration satellite, the primary orbit design method of the nano calibration satellite is established and validated through STK. The error factors of the space-based method is analyzed and simulation work is performed to verify that the accuracy of this space-based method is within $0.3 \mathrm{~dB}$. Compared with the traditional ground-based calibration technologies, the new space-based method using nano calibration satellites can break the limitations of the ground calibration sites, and may provide a low-cost and promising solution for SAR calibration in the future.

Author Contributions: J.H. played the leading role in preparing this paper. T.Q. performed the simulations and analyzed the data. Y.W. contributed to the structure and revision of this paper and provided insightful comments and suggestions. S.D., K.X. and J.M. provided valuable suggestions and revised the manuscript. All authors have read and agreed to the published version of the manuscript.

Funding: This research was funded by the National Natural Science Foundation of China (No. 61771453).

Institutional Review Board Statement: Not applicable.

Informed Consent Statement: Not applicable.

Data Availability Statement: Not applicable.

Conflicts of Interest: The authors declare no conflict of interest.

\section{References}

1. Matar, J.; Rodriguez-Cassola, M.; Krieger, G.; López-Dekker, P.; Moreira, A. MEO SAR: System Concepts and Analysis. IEEE Trans. Geosci. Remote. Sens. 2020, 58, 1313-1324. [CrossRef]

2. Matar, J.; Rodriguez-Cassola, M. Mission Considerations for Future MEO SAR Systems. In Proceedings of the 12th European Conference on Synthetic Aperture Radar, EUSAR 2018, Aachen, Germany, 4-7 June 2018; pp. 1-5.

3. Freeman, A. SAR CALIBRATION-AN OVERVIEW. IEEE Trans. Geosci. Remote. Sens. 1992, 30, 1107-1121. [CrossRef]

4. Shimada, M.; Freeman, A. A technique for measurement of spaceborne SAR antenna patterns using distributed targets. IEEE Trans. Geosci. Remote. Sens. 1995, 33, 100-114. [CrossRef]

5. Seifert, P.; Lentz, H.; Zink, M.; Heel, F. Ground-based measurements of inflight antenna patterns for imaging radar systems. IEEE Trans. Geosci. Remote. Sens. 1992, 30, 1131-1136. [CrossRef]

6. Lukowski, T.; Hawkins, R.; Cloutier, C.; Wolfe, J.; Teany, L.; Srivastava, S.; Banik, B.; Jha, R.; Adamovic, M. RADARSAT elevation antenna pattern determination. In Proceedings of the 1997 IEEE International Geoscience and Remote Sensing Symposium Proceedings, Remote Sensing_A Scientific Vision for Sustainable Development, IGARSS'97, Singapore, 3-8 August 1997; Volume 3, pp. 1382-1384.

7. Seifert, P. ERS-1 Preliminary Antenna Elevation Pattern Measured by Ground-Receivers; ESA: Paris, France, 1993; pp. 157-159.

8. Thompson, A.; Racine, D.; Luscombe, A. RADARSAT-2 antenna calibration using Ground Receivers/Transmitters. In Proceedings of the IEEE International Geoscience and Remote Sensing Symposium, Toronto, ON, Canada, 24-28 June 2002; Volume 3, pp. 1465-1467.

9. Lenz, R.; Pontes, J.; Wiesbeck, W. A high accuracy calibration and receive instrument for TerraSAR-X ground calibration. In Proceedings of the European Radar Conference, EURAD 2005, London, UK, 6-7 October 2005; pp. 411-414. 
10. Lenz, R.; Pontes, J.; Wiesbeck, W. The TerraSAR-X ground calibration system and pattern estimation software. In Proceedings of the 2005 IEEE International Geoscience and Remote Sensing Symposium, IGARSS '05, Seoul, Korea, 29 July 2005; Volume 7, pp. 4894-4897.

11. Fang, Y.; Moore, R. Inflight vertical antenna patterns for SIR-C from Amazon rain-forest observations. In Proceedings of the IGARSS '96, 1996 International Geoscience and Remote Sensing Symposium, Lincoln, NE, USA, 31 May 1996; Volume 1, pp. 496-498.

12. Schwerdt, M.; Brautigam, B.; Bachmann, M.; Doring, B.; Schrank, D.; Hueso Gonzalez, J. Final TerraSAR-X Calibration Results Based on Novel Efficient Methods. IEEE Trans. Geosci. Remote. Sens. 2010, 48, 677-689. [CrossRef]

13. Bachmann, M.; Schwerdt, M.; Brautigam, B. TerraSAR-X Antenna Calibration and Monitoring Based on a Precise Antenna Model. IEEE Trans. Geosci. Remote. Sens. 2010, 48, 690-701. [CrossRef]

14. Brautigam, B.; Schwerdt, M.; Bachmann, M. An Efficient Method for Performance Monitoring of Active Phased Array Antennas. IEEE Trans. Geosci. Remote. Sens. 2009, 47, 1236-1243. [CrossRef]

15. Reimann, J.; Schwerdt, M.; Schmidt, K.; Ramon, N.T.; Döring, B. The DLR Spaceborne SAR Calibration Center. Frequenz 2017, 71, 619-627. [CrossRef]

16. Schwerdt, M.; Schmidt, K.; Tous Ramon, N.; Klenk, P.; Yague-Martinez, N.; Prats-Iraola, P.; Zink, M.; Geudtner, D. Independent System Calibration of Sentinel-1B. Remote. Sens. 2017, 9, 511. [CrossRef]

17. Edelstein, W.; Madsen, S.; Moussessian, A.; Chen, C. Concepts and Technologies for Synthetic Aperture Radar from MEO and Geosynchronous orbits. In Proceedings of the SPIE-The International Society for Optical Engineering, San Diego, CA, USA, 30 September 2005.

18. Guccione, P.; Scagliola, M.; Giudici, D. Low-Frequency SAR Radiometric Calibration and Antenna Pattern Estimation by Using Stable Point Targets. IEEE Trans. Geosci. Remote. Sens. 2018, 56, 635-646. [CrossRef]

19. Prosser, R. The Lincoln calibration sphere. Proc. IEEE 1965, 53, 1672-1673. [CrossRef]

20. Langer, J.V.; Feess, W.A.; Hanington, K.M.; Bacigalupi, M.R.; Cardoza, M.A.; Mach, R.G.; Abusali, P. RADCAL: Precision orbit determination with a commercial grade GPS receiver. In Proceedings of the 1994 National Technical Meeting of The Institute of Navigation, San Diego, CA, USA, 24-26 January 1994; pp. 421-431.

21. Fox, N.; Green, P. Traceable Radiometry Underpinning Terrestrial- and Helio-Studies (TRUTHS): An Element of a Space-Based Climate and Calibration Observatory. Remote. Sens. 2020, 12, 2400. [CrossRef]

22. Fox, N.P.; Green, P.D.; Winkler, R.; Lobb, D.; Friend, J. Traceable Radiometery Underpinning Terrestrial- and Helio- Studies (TRUTHS): Establishing a climate and calibration observatory in space. In Proceedings of the 2016 IEEE International Geoscience and Remote Sensing Symposium (IGARSS), Beijing, China, 10-15 July 2016; pp. 1939-1942. [CrossRef]

23. Tobin, D.; Holz, R.; Nagle, F.; Revercomb, H. Characterization of the Climate Absolute Radiance and Refractivity Observatory (CLARREO) ability to serve as an infrared satellite intercalibration reference. J. Geophys. Res. Atmos. 2016, 121, $4258-4271$. [CrossRef]

24. Shea, Y.; Fleming, G.; Kopp, G.; Lukashin, C.; Pilewskie, P.; Smith, P.; Thome, K.; Wielicki, B.; Liu, X.; Wu, W. Clarreo Pathfinder: Mission Overview and Current Status. In Proceedings of the IGARSS 2020-2020 IEEE International Geoscience and Remote Sensing Symposium, Waikoloa, HI, USA, 26 September-2 October 2020; pp. 3286-3289. [CrossRef]

25. Yu, W.; Hong, J. Potentials of SAR Calibration Satellite Network in Spaceborne SAR External Calibration. In Proceedings of the EUSAR 2018, 12th European Conference on Synthetic Aperture Radar, Aachen, Germany, 4-7 June 2018; pp. 1-4.

26. Shiroma, W.A.; Martin, L.K.; Fisher, N.G.; Jones, W.H.; Furumo, J.G.; Heong, J.R.A.; Umeda, M.M. Ho'oponopono: A Radar Calibration CubeSat. 2011. Available online: https: / digitalcommons.usu.edu/cgi /viewcontent.cgi?filename $=0 \& a r t i c l e=1138$ \&context $=$ smallsat\&type $=$ additional (access date 28 December 2021).

27. Ma, H.; Stove, A.G.; Atkinson, G.; Underwood, C.I.; Cherniakov, M.; Antoniou, M. Passive SAR using small satellite receivers for persistent earth observation. In Proceedings of the International Conference on Radar Systems (Radar 2017), Jakarta, Indonesia, 23-26 October 2017; pp. 1-6.

28. Atkinson, G.; Sayin, A.; Underwood, C.I.; Cherniakov, M.; Antoniou, M. Passive SAR Satellite System (PASSAT): First airborne trials. In Proceedings of the 2019 20th International Radar Symposium (IRS), Ulm, Germany, $26-28$ June 2019; pp. 1-7.

29. Raney, R. Considerations for SAR image quantification unique to orbital systems. IEEE Trans. Geosci. Remote. Sens. 1991, 29, 754-760. [CrossRef] 